\title{
Silencing long non-coding RNA Kcnq1ot1 alleviates pyroptosis and fibrosis in diabetic cardiomyopathy
}

Fan Yang ${ }^{1}$, Ying Qin², Jie Lv' ${ }^{1}$, Yueqiu Wang ${ }^{1}$, Hui Che ${ }^{1,2}$, Xi Chen ${ }^{3}$, Yanan Jiang ${ }^{2,3}$, Anqi Li $^{3}$, Xi Sun ${ }^{3}$, Er Yue ${ }^{3}$, Long Ren ${ }^{3}$, Yang Li', Yunlong Bai, ${ }^{2,3}$ and Lihong Wang ${ }^{1,2}$

\begin{abstract}
Diabetes cardiomyopathy (DCM) is a critical complication of long-term chronic diabetes mellitus and is characterized by myocardial fibrosis and myocardial hypertrophy. It has been suggested that DCM is related to pyroptosis, a programmed cell death associated with inflammation. The long non-coding RNA Kcnq1ot1 is involved in different pathophysiological mechanisms of multiple diseases, including acute myocardial damage and arrhythmia. Our previous study found that Kcnq1ot1 was elevated in left ventricular tissue of diabetic mice. However, whether Kcnq1ot1 is capable of regulating pyroptosis and fibrosis in high glucose-treated cardiac fibroblasts remains unknown. The aim of the study was to investigate the mechanisms of Kcnq1ot1 in DCM. Our study revealed that silencing Kcnq10t1 by a lentivirus-shRNA improved cardiac function and fibrosis, ameliorated pyroptosis, and inhibited TGF- $\beta 1 /$ smads pathway in C57BL/6 mice. In vitro, experiments revealed that Kcnq1ot1 and pyroptosis were activated in cardiac fibroblasts treated with $30 \mathrm{mmol} / \mathrm{l}$ glucose. Furthermore, Kcnq1ot1 knockdown by a small interfering RNA decreased caspase-1 expression. Bioinformatic prediction and luciferase assays showed that Kcnq1 ot1 functioned as a competing endogenous RNA to regulate the expression of caspase-1 by sponging miR-214-3p. In addition, silencing Kcnq1ot1 promoted gasdermin $D$ cleavage and the secretion of $\mathrm{IL}-1 \beta$, thus repressing the TGF- $\beta 1 / \mathrm{smads}$ pathway in high glucose-treated cardiac fibroblasts through miR-214-3p and caspase-1. Therefore, Kcnq1 ot1/miR-214-3p/caspase-1/ TGF- $\beta 1$ signal pathway presents a new mechanism of DCM progression and could potentially be a novel therapeutic target.
\end{abstract}

\section{Introduction}

Diabetic cardiomyopathy (DCM) is a vital complication of diabetes mellitus (DM) and is characterized by structural and functional dysfunction in the absence of hypertension, coronary artery disease, and other cardiac pathologies ${ }^{1}$. It has been suggested that cell death,

\footnotetext{
Correspondence: Lihong Wang (nd6688@163.com) or

Yunlong Bai (baiyunlong@ems.hrbmu.edu.cn)

'Department of Endocrinology, The Second Affiliated Hospital of Harbin Medical University, Harbin, China

${ }^{2}$ Translational Medicine Research and Cooperation Center of Northern China, Heilongjiang Academy of Medical Sciences, Harbin, China

Full list of author information is available at the end of the article.

These authors contributed equally: Fan Yang, Ying Qin.

Edited by B. Rotblat
}

mitochondrial dysfunction, and inflammation are all hallmarks of cardiac hypertrophy and myocardial fibrosis. The fibrosis is the characteristic pathological manifestation of DCM and enhances the risk of heart failure, arrhythmia and sudden death ${ }^{2}$. Therefore, the study of the mechanisms underlying myocardial fibrosis is essential.

Pyroptosis is a type of programmed cell death related to the activation of inflammation ${ }^{3}$. It has been reported that hyperglycemia activates nucleotide-binding oligomerization domain-like receptor pyrin domain containing (NLRP) 3 inflammasome, thus promoting procaspase- 1 to caspase- 1 . The cleaved caspase- 1 is then

\section{(c) The Author(s) 2018}

(c) (i) Open Access This article is licensed under a Creative Commons Attribution 4.0 International License, which permits use, sharing, adaptation, distribution and reproduction c. in any medium or format, as long as you give appropriate credit to the original author(s) and the source, provide a link to the Creative Commons license, and indicate if changes were made. The images or other third party material in this article are included in the article's Creative Commons license, unless indicated otherwise in a credit line to the material. If material is not included in the article's Creative Commons license and your intended use is not permitted by statutory regulation or exceeds the permitted use, you will need to obtain permission directly from the copyright holder. To view a copy of this license, visit http://creativecommons.org/licenses/by/4.0/. 
capable of converting the pro-interleukin-1 $\beta$ (pro-IL$1 \beta$ ) and pro-interleukin-18 (pro-IL-18) into matured IL- $1 \beta / \mathrm{IL}-18^{4}$. Recently, studies have revealed that gasdermin D (GSDMD) is another critical component of the inflammasome and can be cleaved by activated caspase- $1^{5,6}$. The cleaved $N$-terminal of GSDMD (GSDMD-N) is essential in the process of pyroptosis because it can promote the secretion of matured IL-1 $\beta$ and damage the plasma membrane ${ }^{6}$. More importantly, to our knowledge, the activation of the inflammasome and the release of cytokines can promote the deposition of collagens and fibrotic formation, further exacerbating the severity of $\mathrm{DCM}^{7}$. Previous studies have shown that the inhibition of pyroptosis by microRNA-9 (miR-9), miR-30d, NLRP3 gene silencing, and rosuvastatin etc. can improve the cardiac function of diabetic mice ${ }^{8-11}$. However, a comprehensive study focused on the molecular mechanisms underlying pyroptosis in cardiac fibroblasts and its effect on cardiac fibrosis is still lacking.

Long non-coding RNAs (lncRNAs) are a group of nonprotein coding RNA transcripts of length $>200$ nucleotides ${ }^{12}$. LncRNAs regulate gene expression in epigenetic, transcriptional, and post-transcriptional levels. LncRNAs have been found to play critical roles in a variety of biological processes, such as cell proliferation and migration, inflammation, apoptosis, and autophagy $^{13}$. The KCNQ1 opposite strand/antisense transcript 1 (Kcnq1ot1) is a lncRNA located in human chromosome $11 \mathrm{p} 15.5^{14}$. A growing number of studies have shown that Kcnq1ot1 is involved in various diseases, including acute myocardial damage and arrhythmia ${ }^{15,16}$. Jin et al. ${ }^{17}$ showed that Kcnq1ot1 could regulate caspase- 1 expression by targeting miR214-3p to promote cataract formation. However, the role and molecular regulatory mechanism of lncRNA Kcnq1ot1 in high glucose (HG)-induced pyroptosis and fibrosis is still unknown. Thus, the present study aimed to probe the ability of Kcnq1ot1 to modulate pyroptosis and cardiac fibrosis in the process of DCM.

In this study, we report for the first time that the IncRNA Kcnq1ot1 was significantly upregulated in diabetic myocardial tissues and HG-treated cardiac fibroblasts. Further investigations demonstrated that Kcnq1ot1 could act as a competitive endogenous RNA (ceRNA) for miR-214-3p to regulate the expression of caspase-1. Knockdown of Kcnq1ot1 ameliorated pyroptosis and fibrosis in vivo and in vitro.

\section{Results}

\section{Silencing Kcnq1 ot 1 ameliorates cardiac function and fibrosis in diabetic mice}

In the pre-experiment, three different siRNAs against Kcnq1ot1 were used to assess the efficiency of Kcnq1ot1 knockdown in cardiac fibroblasts. The results showed that the expression levels of Kcnq1ot1 were significantly decreased after transfection with all three siRNAs (Supplementary Figure 1a). The si-Kcnq1ot1-1 with the better efficiency at silencing Kcnq1ot1 expression was selected for the subsequent experiments. The lentivirus carrying shRNA targeting Kcnq1ot1 was constructed based on the sequence of si-Kcnq1ot1-1.

To investigate the potential function of Kcnq1ot1 in DCM, we established a streptozotocin (STZ)-induced diabetic model in C57BL/6 mice. As shown in Fig. 1a, Kcnq1ot1 expression was dramatically elevated in cardiac tissue of the diabetic mice and downregulated after injection with the Kcnq1ot1-shRNA lentivirus. Functionally, the echocardiographic data indicated a deterioration of the systolic and diastolic functions of the left ventricular, and the ejection fraction (EF) and fractional shortening (FS) were decreased in the DM group. However, these changes were restored to some extent after treatment with Kcnq1ot1-shRNA (Fig. 1b-d). Morphologically, hematoxylin and eosin stain (HE), and Masson's trichrome staining revealed that the increased myocardial mass and collagen deposition induced in the diabetic heart were ameliorated after silencing Kcnq1ot1 expression (Fig. 1e). Furthermore, western blot analysis showed that collagen I and collagen III were significantly reduced after silencing Kcnq1ot1 (Fig. 1f). TGF- $\beta 1$, phosphorylated smad2 (p-smad2) and p-smad3 expression levels were remarkably increased in the DM group; however, TGF- $\beta 1 /$ smads signaling pathway was significantly inhibited in the DM+Kcnq1ot1-shRNA group (Fig. 1g).

\section{Kcnq1ot 1 is involved in the regulation of pyroptosis in vivo} Immunohistochemistry analysis indicated that NLRP3, caspase-1, IL-1 $\beta$, and GSDMD-N levels were significantly elevated in cardiac tissue of diabetic mice. However, the levels were reduced after Kcnq1ot1 silencing (Fig. 2a). The mRNA and protein expression levels of NLRP3, caspase-1, and IL- $1 \beta$ were remarkably increased in the DM group and were reduced after treatment with Kcnq1ot1-shRNA (Fig. 2b-e). Moreover, protein expression levels of GSDMD-N were significantly increased in the diabetic mice, and were reversed following Kcnq1ot1 inhibition (Fig. 2e). These data demonstrate that lncRNA Kcnq1ot1 modulates pyroptosis in the diabetic heart.

\section{Kcnq1ot1 and pyroptosis are activated in HG-treated cardiac fibroblasts}

We further confirmed the effects of Kcnq1ot1 on pyroptosis with in vitro experiments. Cardiac fibroblasts of 
a

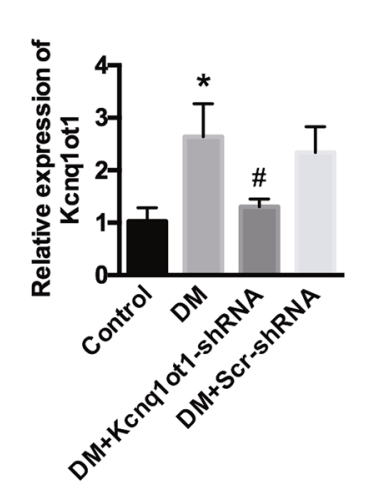

b

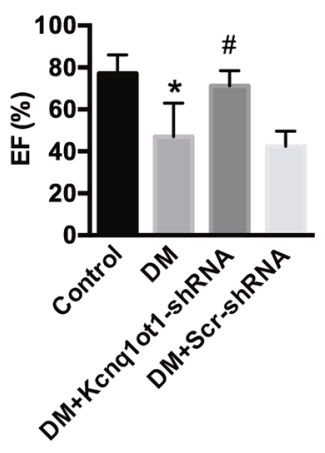

C
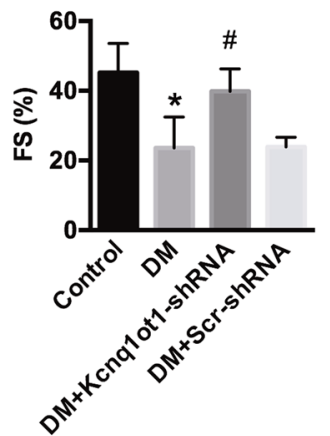

d
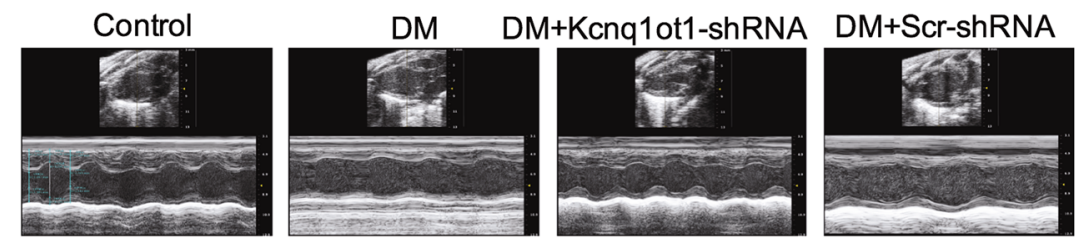

e
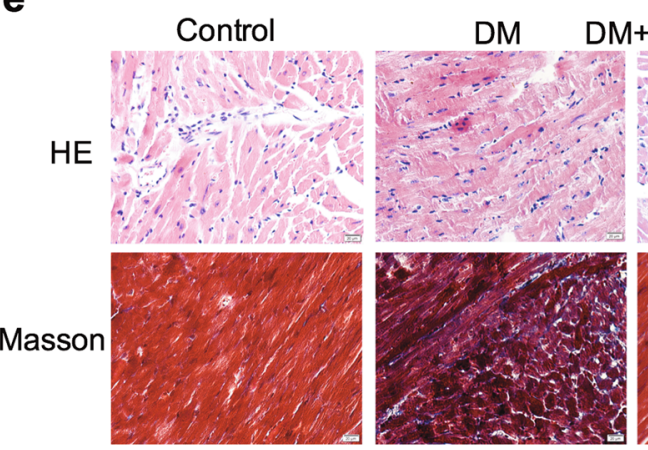

$D M+K c n q 1$ ot1-shRNA

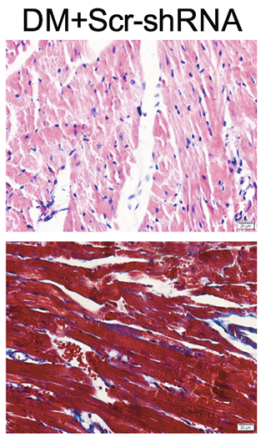

f

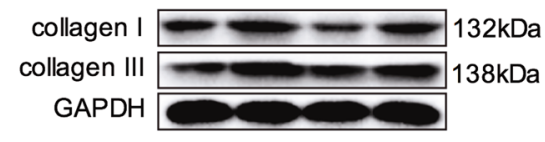

g

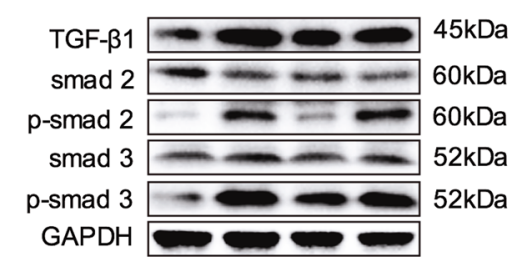

Control

DM

DM+Kcnq1ot1-shRNA DM+Scr-shRNA
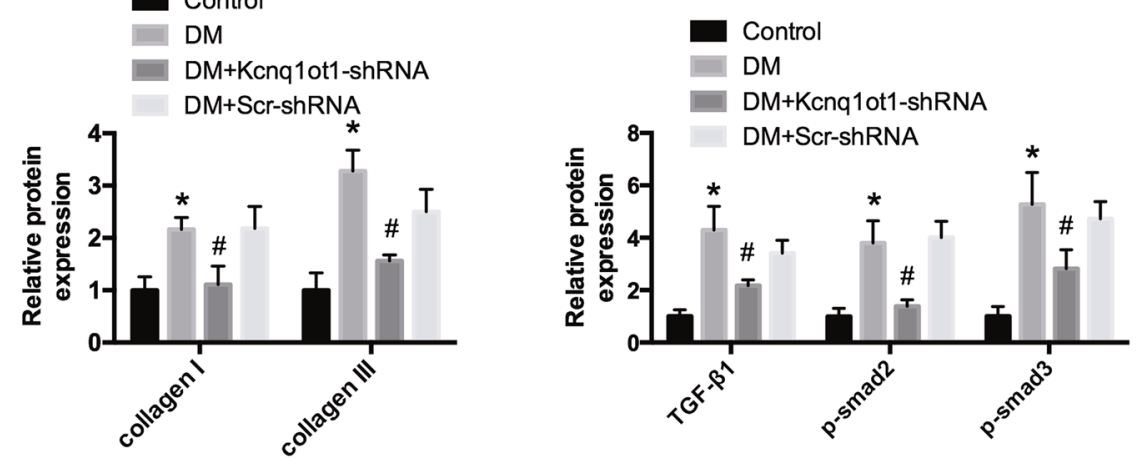

Fig. 1 Silencing Kcnq1ot1 attenuates cardiac function and structure in diabetic mice a The expression of Kcnq1 ot 1 in left ventricle was detected by qRT-PCR. M-mode echocardiograms of left ventricle $\mathbf{b}$, ejection fraction (EF) $\mathbf{c}$ and shortening fraction (FS) $\mathbf{d}$ are shown. e Hematoxylineosin (HE) and Masson's trichrome staining were performed. Scale bar, $50 \mu \mathrm{m}$. $\mathbf{f}$ The expression levels of collagen I and collagen III were detected by western blot. $\mathbf{g}$ Relative protein expression of TGF- $\beta 1$, $p$-smad2, and $p$-smad3 were detected by western blot. ${ }^{*}<<0.05$ compared with the control group, ${ }^{\#} P<0.05$ compared with the DM + Scr-shRNA group. $n=5$ in each group 
a

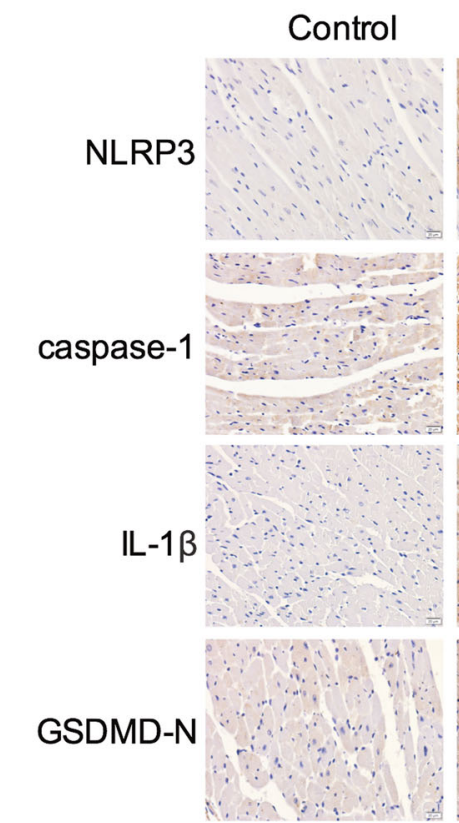

b

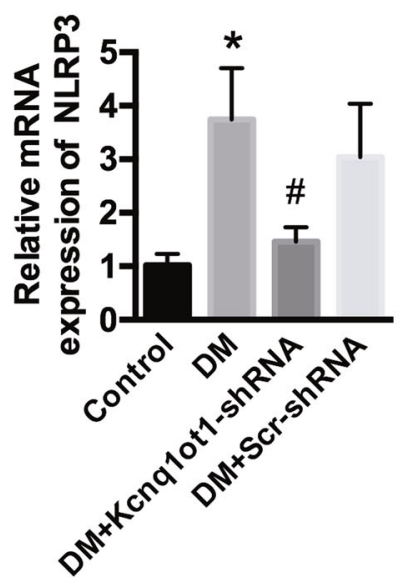

e

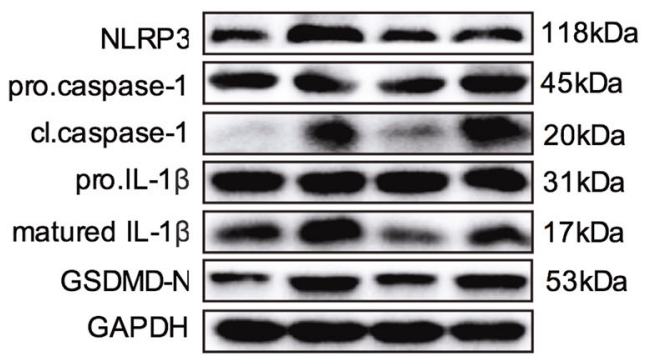

C
DM DM+Kcnq1ot1-shRNA DM+Scr-shRNA
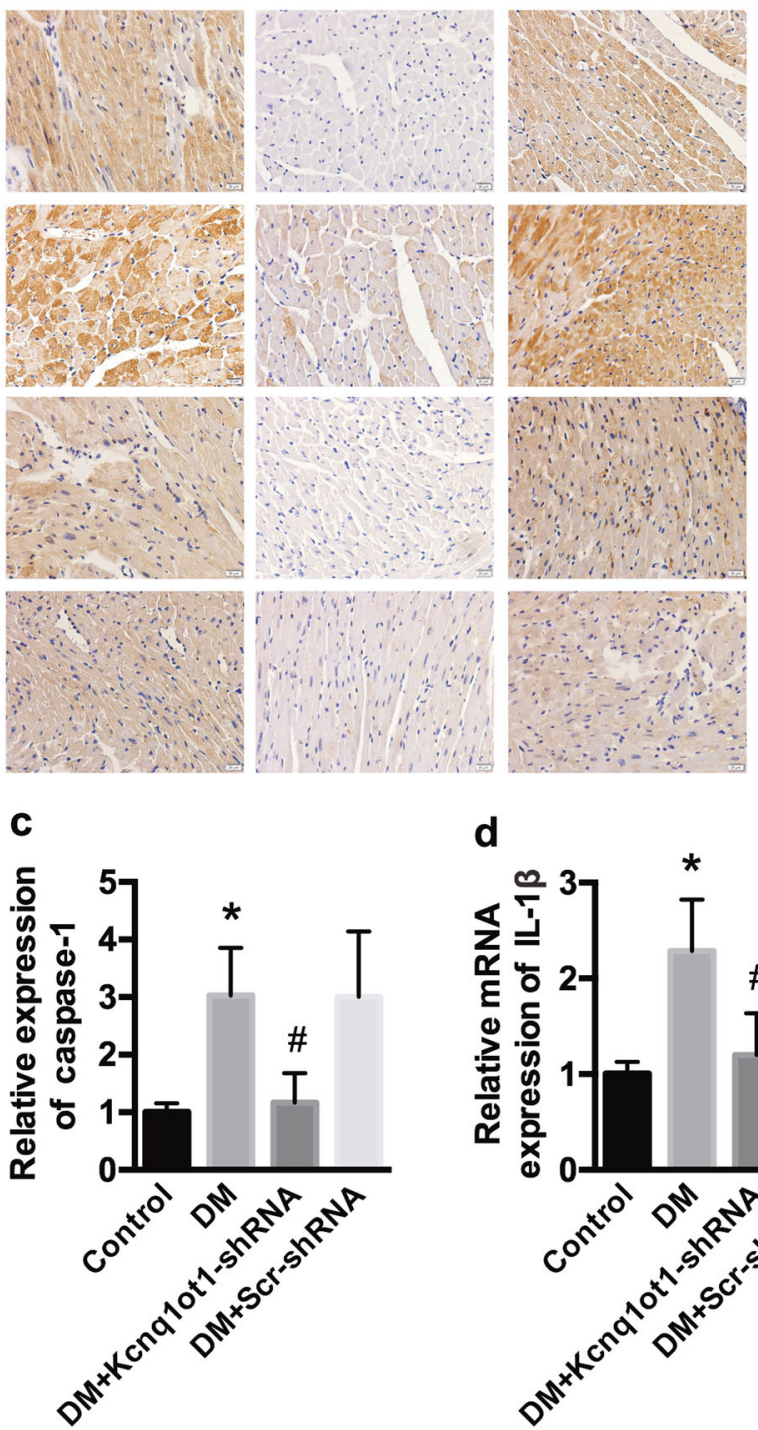

d

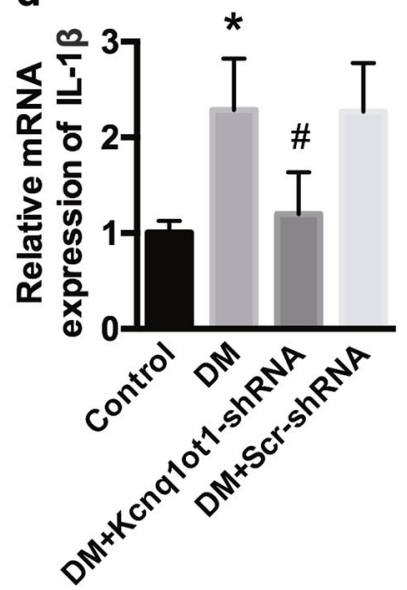

Control

DM

DM+Kcnq1ot1-shRNA

DM+Scr-shRNA

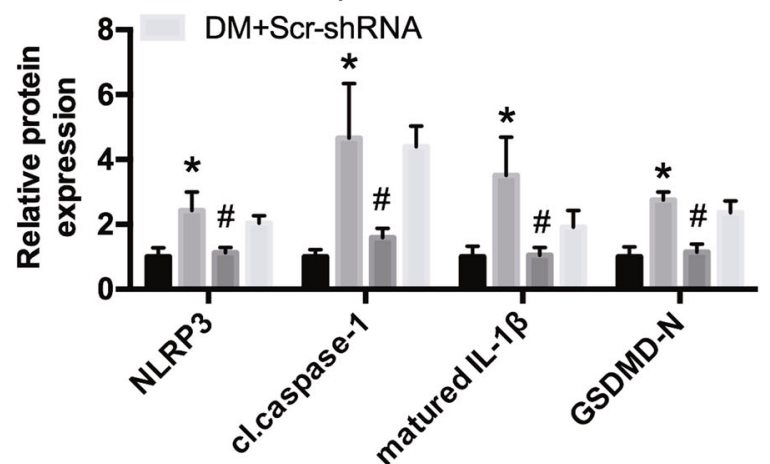

Fig. 2 Kcnq1ot 1 is involved in the regulation of pyroptosis in vivo a Immunohistochemistry analysis was performed to detect the expression NLRP3, caspase-1, IL-1 $\beta$, and GSDMD-N. Scale bar, $20 \mu \mathrm{m}$. b-d qRT-PCR was conducted to analyze the mRNA expression of NLRP3, caspase-1 and IL-1 $\beta$. e Western blot was conducted to determine the protein expression of NLRP3, caspase- 1 and IL-1 $\beta$. ${ }^{*} P<0.05$ compared with the control group, ${ }^{\#} P<0.05$ compared with the DM + Scr-shRNA group. $n=5$ in each group 


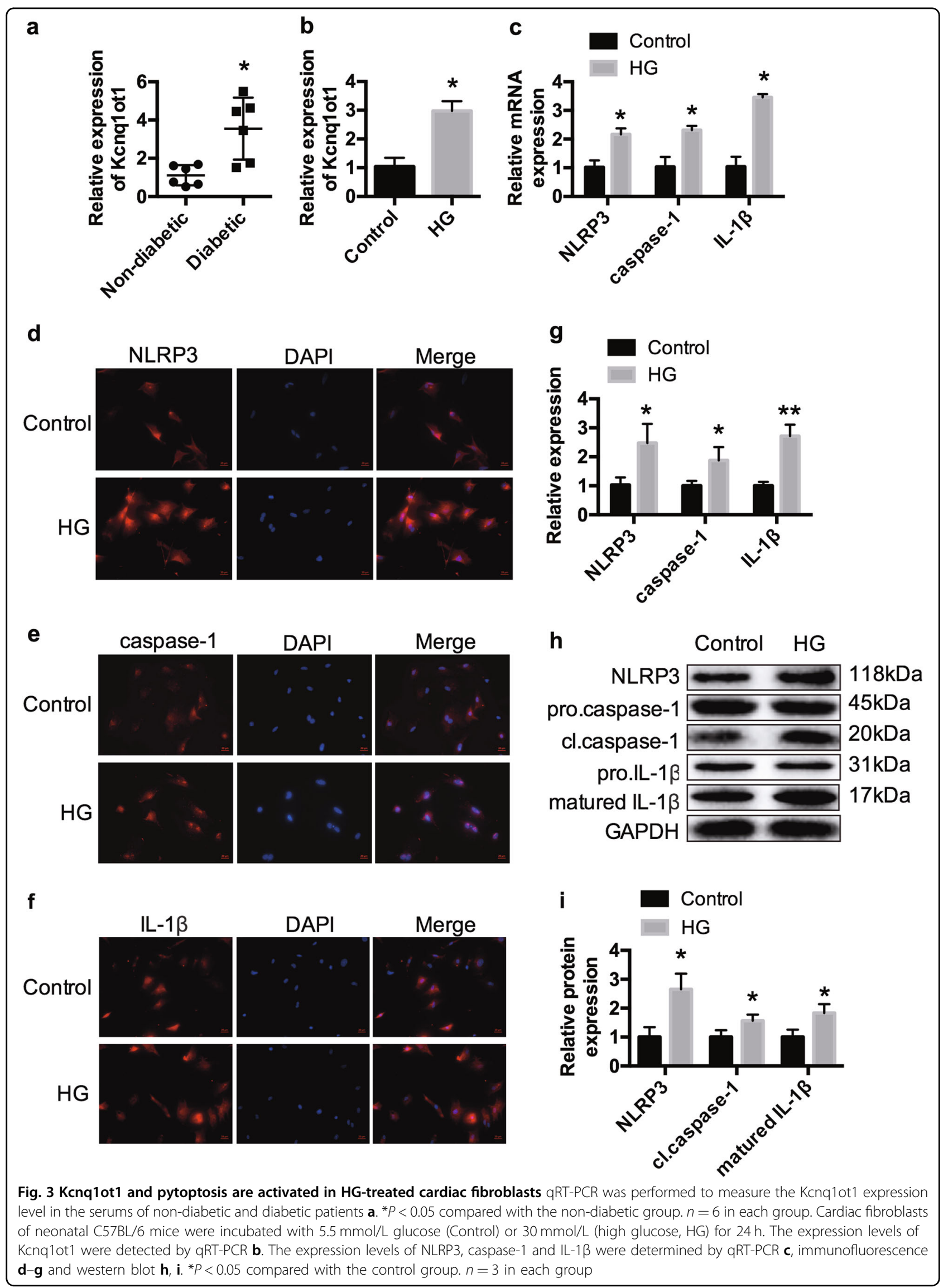


neonatal C57BL/6 mice were incubated with either 5.5 $\mathrm{mmol} / \mathrm{L}$ glucose (Control) or $30 \mathrm{mmol} / \mathrm{L}$ (HG) for $24 \mathrm{~h}$. qRT-PCR revealed that Kcnq1ot1 was significantly elevated both in the serum of diabetic patients and HGtreated cardiac fibroblasts (Fig. 3a, b). Consistent with the in vivo results, the expression levels of NLRP3, caspase-1, and IL- $1 \beta$ were also elevated in the HG-treated cardiac fibroblasts as revealed by qRT-PCR, immunofluorescence, and western blot (Fig. 3c-i).

\section{miR-214-3p contains both Kcnq1 ot 1 and caspase-1- binding sites}

Silencing of Kcnq1ot1 by siRNA in the HG group (Fig. 4a) led to an evident decrease in the mRNA and protein expression levels of caspase-1 (Fig. 4b, c). In addition, the expression levels of caspase-1 were also downregulated after silencing Kcnq1ot1 with different siRNAs (Supplementary Figure 1b). We demonstrated that the results of the experiments were not due to the phenomenon of sequence specific off-target-derived phenotypes. Bioinformatic prediction analysis indicated that miR-214-3p potentially interacted with both Kcnq1ot1 and caspase-1. The binding sites are shown in Fig. 4d, e. Our team had previously demonstrated the complementary regulatory relationship between Kcnq1ot1 and miR-214-3p, as well as caspase-1 and miR214-3p, by luciferase assays in HEK293T cells ${ }^{17}$. The binding sites of KCNQ1OT1 were cloned into the luciferase vector, and miR-214-3p mimics were cotransfected into HEK293T cells. The luciferase activity was significantly repressed when the cell was cotransfected with miR-214-3p mimics, whereas the mutation of the miR-214-3p target site reversed the repression (Supplementary Figure 2a and b). Moreover, a luciferase construction carrying the 3 '-UTR of caspase- 1 was generated. The results indicated that the translation of wildtype caspase- 1 was inhibited by miR-214-3p mimics, as shown by the reduced luciferase signal, whereas the luciferase activity was unaffected by miR-214-3p mimics when the construction carried the $3^{\prime}$-UTR of caspase-1 with a mutant miR-214-binding site (Supplementary Figure $2 \mathrm{c}$ and $\mathrm{d}$ ). qRT-PCR experiments indicated that miR-214-3p was decreased in the HG-treated cardiac fibroblasts (Fig. 4f), the serums of diabetic patients (Fig. 4g), and the cardiac tissue of diabetic mice (Fig. 4h). Silencing of Kcnq1ot1 resulted in an increased miR-214$3 p$ expression in vivo and in vitro (Fig. $4 \mathrm{~h}$, i). We then performed gain-of-function and loss-of-function experiments to evaluate the effects of miR-214-3p on caspase-1 in fibroblasts. Fig. 4j indicates successful transfection. Overexpression of miR-214-3p led to decreased caspase-1 levels, whereas caspase-1 levels were increased after inhibition by miR-214-3p in the HG-treated cardiac fibroblasts (Fig. 4k, l).

\section{Silencing Kcnq1 ot 1 inhibits caspase-1 expression by targeting miR-214-3p in vitro}

In addition, cardiac fibroblasts incubated with $5.5 \mathrm{mmol} / \mathrm{L}$ glucose were transfected with si-NC and AMO-NC. Cells incubated with $\mathrm{HG}$ were divided into three transfection groups: those transfected with control si-NC and AMO$\mathrm{NC}$, si-Kcnq1ot1 and AMO-NC, and si-Kcnq1ot1 and AMO-214-3p. The expression levels of Kcnq1ot1 and miR214-3p are shown in Fig. 5a, b. qRT-PCR indicated that the expression of caspase-1 was increased in the HG group and were significantly decreased after being transfected with siKcnq1ot1, whereas the inhibition was attenuated by cotransfection with AMO-214-3p (Fig. 5c). Simultaneously, immunofluorescence staining and western blot analysis also confirmed these results (Fig. 5d, e). Above all, we confirm that Kcnq1ot1 regulates caspase-1 expression by miR-214$3 p$ in cardiac fibroblasts.

\section{Kcnq1ot1 regulates inflammation and pyroptosis of cardiac fibroblasts by sponging miR-214-3p}

Cardiac fibroblasts were transfected with si-Kcnq1ot1 with or without AMO-214-3p in HG conditions. We observed that HG promoted increased NLRP3 and IL-1 $\beta$ expression, whereas these levels were decreased after silencing Kcnq1ot1. However, co-transfection with siKcnq1ot1 and AMO-214-3p promoted NLRP3 and IL-1 $\beta$ expression (Fig. 6a-f). In addition, western blot analysis was conducted to detect the expression of GSDMD-N. The results showed that GSDMD-N was evidently elevated in the HG-treated fibroblasts and was decreased after silencing Kcnq1ot1. However, the repressive effect was abrogated by AMO-214-3p (Fig. 6g). Therefore, silencing Kcnq1ot1 suppresses inflammation and pyroptosis by inhibiting the Kcnq1ot1/miR-214-3p pathway in HG-treated cardiac fibroblasts.

\section{Silencing Kcnq1ot1 alleviates fibrosis of HG-induced fibroblasts}

Previous studies revealed that HG could increase collagen I and collagen III expressions, and activate TGF- $\beta 1$, $\mathrm{p}$-smad2, and p-smad3. Here, we found that collagen I and collagen III expressions as well as the activated TGF$\beta 1 /$ smads pathway were repressed after silencing Kcnq1ot1, and were reversed by co-transfection with AMO-214-3p (Fig. 7). Therefore, we suggest that silencing Kcnq1ot1 alleviates inflammation and fibrosis of HGinduced fibroblasts through miR-214-3p.

\section{Discussion}

DCM is an important cardiovascular complication of diabetes, but its pathogenesis is not yet fully elucidated. It has been documented that IncRNAs play vital roles in many diseases. The aim of the study was to investigate the biological function of the lncRNA Kcnq1ot1 in the 


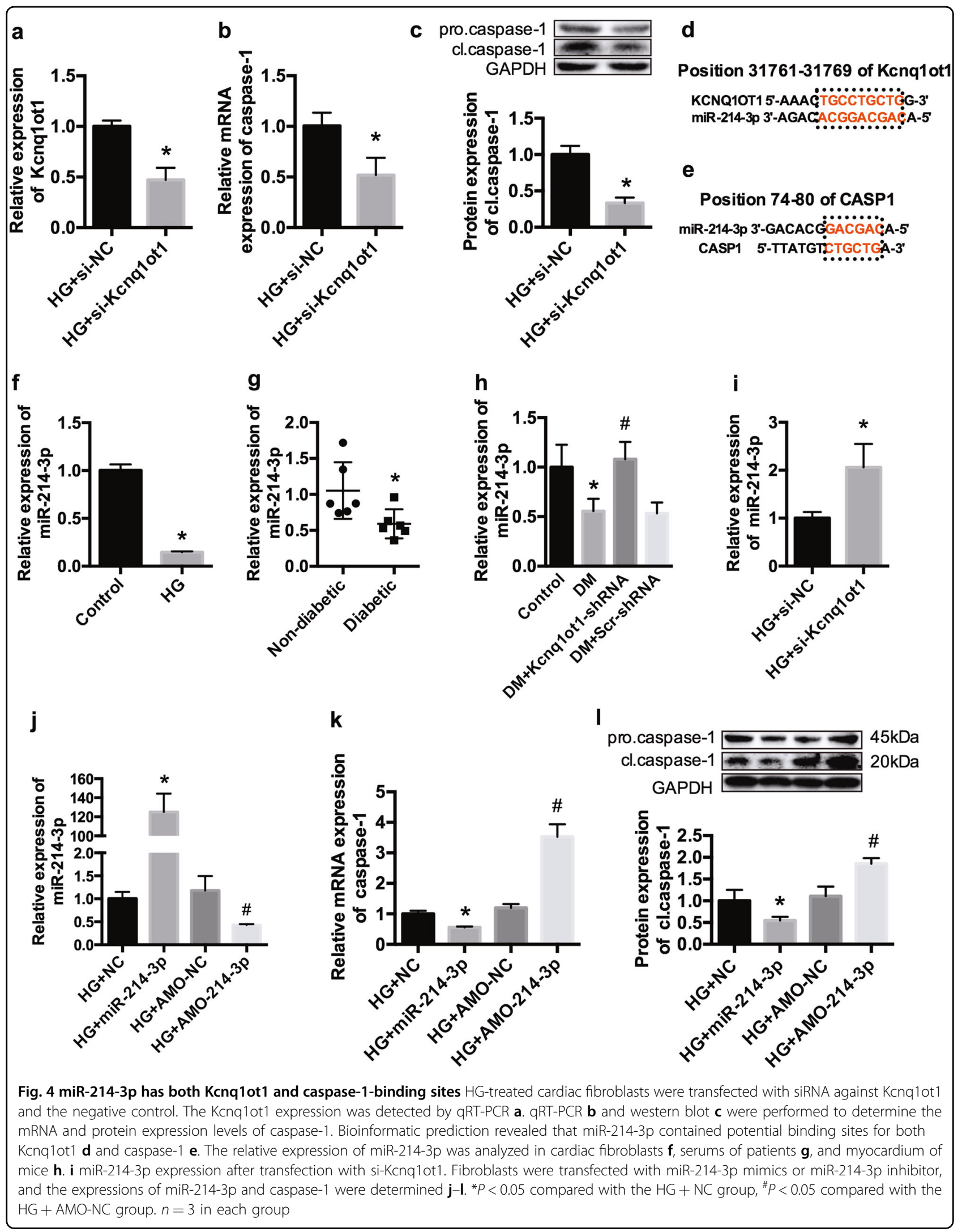




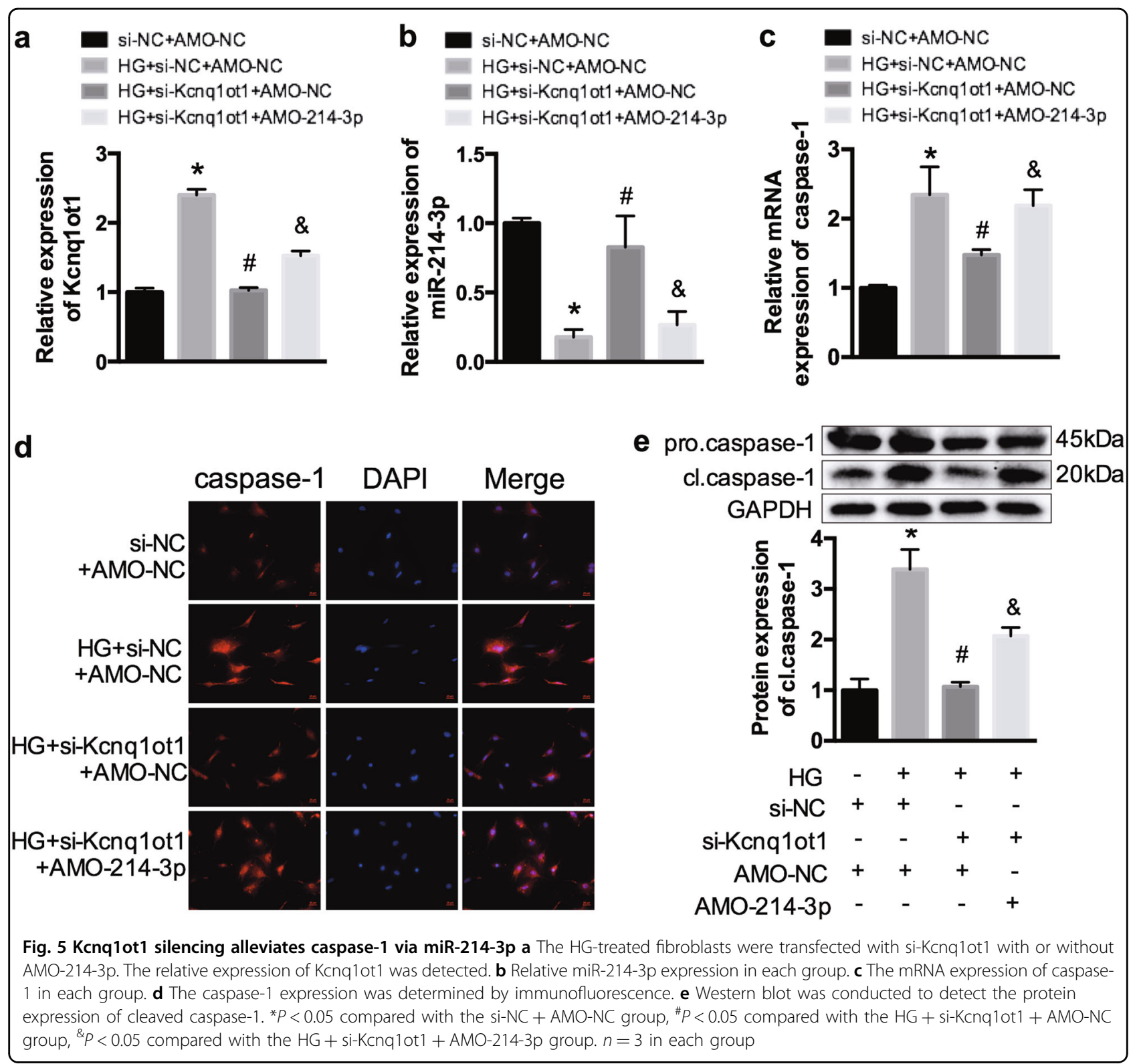

development and procession of DCM. A critical finding of our research is that Kcnq1ot1 is overexpressed in DCM. Further, silencing Kcnq1ot1 alleviates myocardial dysfunction and attenuates myocardial fibrosis in STZinduced C57BL/6 mice. In vitro studies revealed that silencing Kcnq1ot1 reduced caspase-1 expression by acting as an endogenous miR-214-3p sponge, thereby repressing downstream cytokines and leading to alleviation of HG-induced inflammation and fibrosis in cardiac fibroblasts. Thus, our study demonstrates the vital role of Kcnq1ot1 in DCM and establishes the lncRNA as a novel therapeutic target of DCM.

LncRNA Kcnq1ot1 has been shown to be closely related to a variety of diseases, including long QT syndrome, cataracts, cancers, and myocardial ischemia/reperfusion injury ${ }^{14-18}$. Recently, Gao et al. ${ }^{14}$ reported that Kcnq1ot1 induced macrophage polarization and improved osteolysis by inhibiting miR-21a-5p. In addition, Guo et al. ${ }^{19}$ found that Kcnq1ot1 promoted cell proliferation and metastasis in melanoma by acting as a ceRNA of miR-153. However, the relationship between Kcnq1ot1 and diabetes as well as its complications are rarely reported. Asahara et al. ${ }^{20}$ reported that Kcnq1ot1 was closely connected with pancreatic $\beta$-cell mass and was likely a key factor in the onset of diabetes. In the present study, our results reveal that lncRNA Kcnq1ot1 is highly expressed in myocardial tissues of STZ-induced diabetic mice and HG-treated cardiac fibroblasts. Inhibiting Kcnq1ot1 ameliorated caspase1 expression significantly. We were the first to elucidate the differential expression of Kcnq1ot1 in DCM models. 


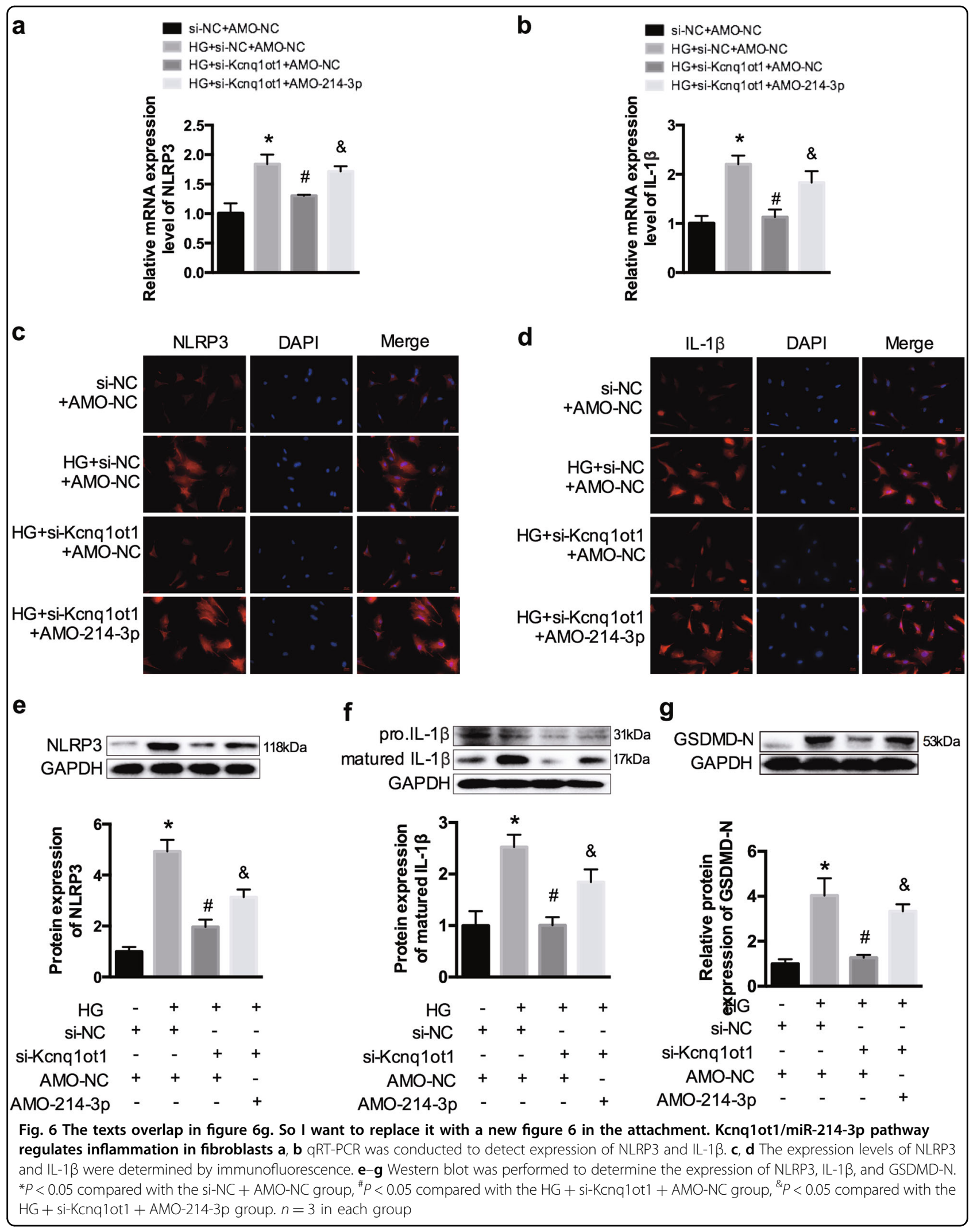




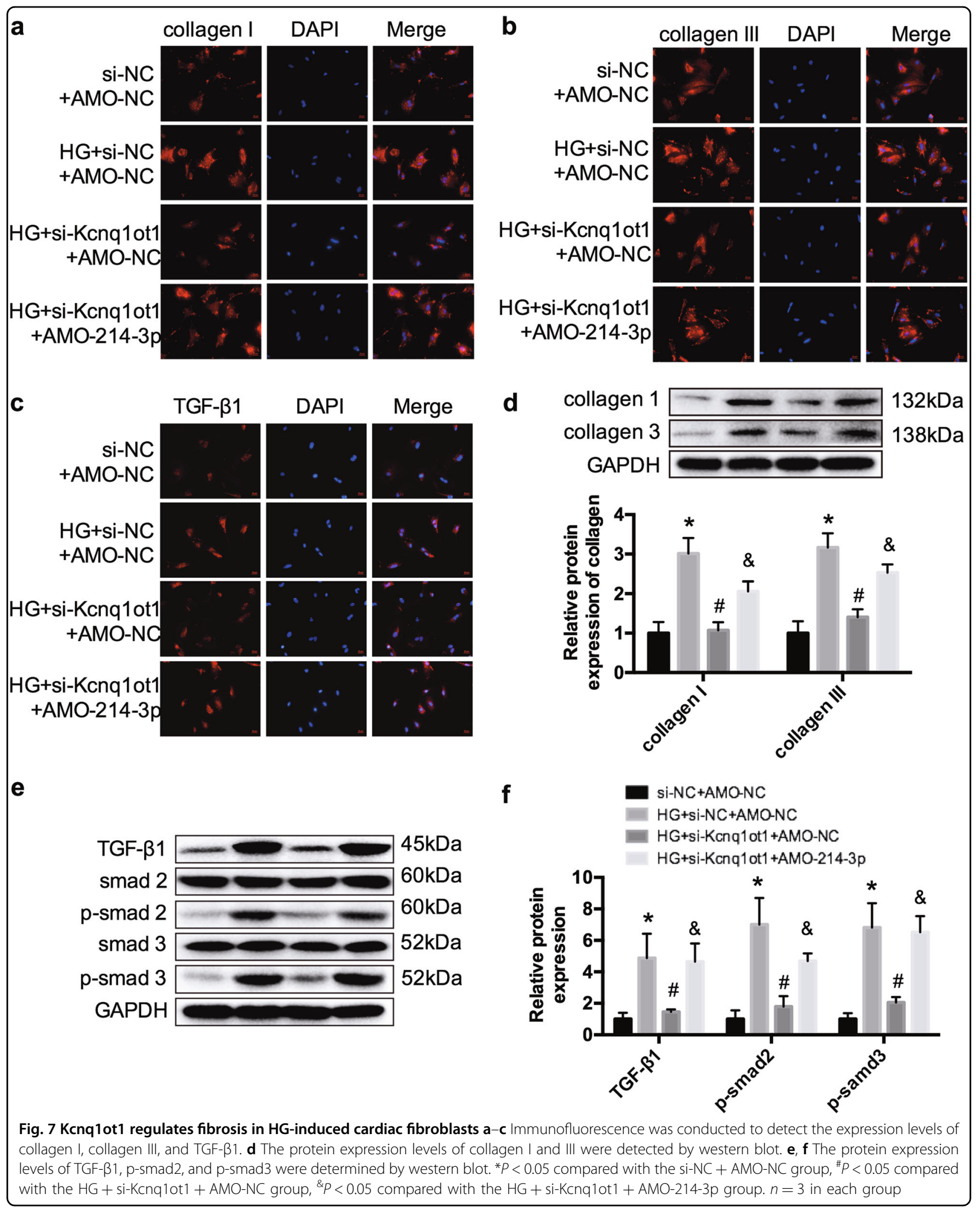


LncRNAs are non-coding RNAs that can regulate the expression of protein-coding messenger RNAs. The ceRNA hypothesis is the most important mechanism of lncRNAs, positing that IncRNA can sponge miRNAs by competing binding sites and regulate gene expres$\operatorname{sion}^{13,21,22}$. The novel RNA cross-talk hypothesis provides a novel method of understanding the mechanisms of various disorders at the post-transcriptional level. In our study, using bioinformatic prediction, miR-214-3p was found to have both Kcnq1ot1 and caspase-1-binding sites, which was consistent with previous study in cataract ${ }^{17}$. Jin et al. have already demonstrated the complementary regulatory relationship among miR-214-3p, Kcnq1ot1 and caspase-1 using luciferase assay. Here, we performed functional assays and proved that Kcnq1ot1 can regulate the expression of caspase- 1 by targeting miR-214-3p in HG-treated cardiac fibroblasts. Therefore, we suggest that Kcnq1ot1 might be an innovative therapeutic target for DCM through its regulation of caspase-1.

Pyroptosis is programmed cell death associated with inflammation and features pore formation, disruption on the plasma membrane, and cell swelling ${ }^{3}$. It was proven that pyroptosis participated in the process of DCM. Caspase-1 is vital to the regulation of pyroptosis and the maturation of cytokine $^{23}$. In the present study, we reveal that Kcnq1ot1 can accelerate pyroptosis of HG-induced fibroblasts by enhancing caspase-1 expression in DCM. After silencing Kcnq1ot1, caspase- 1 and its downstream inflammatory cytokines, IL-1 $\beta$, were repressed remarkably via miR-214-3p targeting. It is worth emphasizing that previous studies have found that IL-1 $\beta$, which is the central link in a variety of pathological processes, is closely related to cardiac fibrosis ${ }^{24-26}$. It was demonstrated that long exposure in IL- $1 \beta$ activated the TGF- $\beta 1$ pathway and induced the transformation of microvascular endothelial cells to myofibroblasts, leading to increased collagen synthesis, promotion of myocardial remodeling and increased interstitial myocardial fibrosis $^{27-29}$. Therefore, we have reason to speculate that silencing Kcnq1ot1 represses collagen deposition and alleviates myocardial fibrosis via inhibition of inflammatory factors. The ceRNA regulatory network, Kcnq1ot1/miR-214$3 \mathrm{p} /$ caspase-1/TGF- $\beta 1$, plays an important role in the regulation of myocardial fibrosis in DCM.

In conclusion, we show that hyperglycemia induces higher expression of Kcnq1ot1 in cardiac fibroblasts. Downregulating Kcnq1ot1 alleviates pyroptosis and fibrosis via the miR-214-3p/caspase-1/TGF- $\beta 1$ pathway. Our study is the first to demonstrate the expression and function of Kcnq1ot1 in DCM and put forward a new method of treatment for DCM based on a non-coding RNA.

\section{Materials and methods}

\section{Serum samples of patients}

The serums were extracted from healthy subjects or diabetic patients in the Second Affiliated Hospital of
Harbin Medical University ( $n=6$ per group). Hypertension, coronary artery disease, and some other heart diseases were precluded in all patients. Patients involved in this research signed an informed consent. All experiments were approved by the ethical committee of Harbin Medical University.

\section{Animal model and treatment}

Male C57BL/6 mice weighing 18-20 g were purchased from the animal experiment center of the Second Affiliated Hospital of Harbin Medical University. Diabetes were induced in the mice by intraperitoneal injection of $50 \mathrm{mg} /$ $\mathrm{kg} /$ day STZ (Sigma, St. Louis, MO, USA) for 5 days. After $72 \mathrm{~h}$, mice with blood glucose of $>16.7 \mathrm{mmol} / \mathrm{L}$ in the tail vein as measured by a Contour glucose meter (Roche, Germany) were considered successful diabetic models $(D M)^{30}$. Diabetic mice were administered with either the Kcnq1ot1 lentivirus-shRNA (DM + Kcnq1ot1-shRNA) or scramble shRNA (DM + Scr-shRNA) (GenePharma, Shanghai, China). The sequence of the shRNA is GGTAG AATAGTTCTGTCTT. Then, $1 \times 10^{9}$ TU lentivirusshRNA was dissolved in $50 \mu \mathrm{L}$ saline and injected into the tail vein of diabetic mice ${ }^{31}$. All mice were kept for 8 weeks and were anesthetized by avertin.

\section{Cell culture and transfection}

The primary cardiac fibroblasts were extracted from hearts of one- to 3-day-old neonatal C57BL/6 mice. The isolation and culture methods were described previously ${ }^{32}$. The cells were cultured with different concentration of glucose: $5.5 \mathrm{mmol} / \mathrm{L}$ glucose (Control) and $30 \mathrm{mmol} / \mathrm{L}$ glucose (HG) for $24 \mathrm{~h}$ at $37^{\circ} \mathrm{C}$ and $5 \% \mathrm{CO}_{2}$ conditions. Cells were transfected with small interfering RNAs (siRNAs) against Kcnq1ot1 (si-Kcnq1ot1), miR-214-3p mimics (miR-214-3p), anti-miRNA oligonucleotides (AMO) of miR-214-3p (AMO-214-3p), or corresponding negative control (si-NC, NC, AMO-NC) designed and synthesized by RIOBIO (Guangzhou, China) for $48 \mathrm{~h}$. The procedure was according to the manufacturer's instructions. The sequences were as follows: si-Kcnq1ot1-1: GGTAGAAT AGTTCTGTCTT; si-Kcnq1ot1-2: GCAGTTATTGAAA CCTCTA; si-Kcnq1ot1-3: CCACATCACAGCAACCT AA; miR-214-3p mimics: forward, 5'-ACAGCAGGCAC AGACAGGCAGU-3', reverse, 3'-UGUCGUCCGUGUCU GUCCGUCA-5'; miR-214-3p inhibitor, 5'-mAmCmUm GmCmCmUmGmUmCmUmGmUmGmCmCmUmGm CmUmGmU-3'.

\section{Echocardiography}

Two-dimensional M-mode echocardiography was detected by a Vevo1100 high-resolution imaging system (VisualSonics, Toronto, ON, Canada) to evaluate the cardiac function. EF and FS of the left ventricle were derived using the machine. 


\section{HE and Masson's trichrome staining}

The cardiac tissues from the left ventricle fixed in $4 \%$ paraformaldehyde were embedded in paraffin and cut into $5 \mu \mathrm{m}$-thick sections. The sections were stained with HE and Masson's trichrome separately. Morphology of the myocardium and deposition of collagen were observed by fluorescence microscope (IX71 Olympus, Japan).

\section{Total RNA isolation and quantitative real-time RT-PCR (qRT-PCR)}

Total RNA in the human serums was extracted by Trizol LS (Invitrogen, USA), and the total RNA of cardiac fibroblasts and cardiac tissues was extracted by Trizol (Invitrogen, USA). RNAs were reversed-transcribed using the ReverTra Ace qPCR RT kit (Code No. FSQ-101, Toyobo, Japan). To detect the expression levels of miR214-3p, we changed the Random Primer Mix to the miR214-3p-specific RT-primers, which were designed by RIOBIO (Guangzhou, China), according to the manufacturer's protocol and a previous study ${ }^{33}$. cDNA was amplified and were detected by an ABI 7500 fast system (Applied Biosystems, CA, USA). U6 was used as internal control for miR-214-3p and GAPDH for all others. The primers sequences are:

Kcnq1ot1 forward: 5'-GCACTCTGGGTCCTGTTCTC-3', Kcnq1ot1 reverse: 5'-CACTTCCCTGCCTCCTACAC-3'; miR-214-3p forward: 5'-TATACATCAAACAGCAGG CACA-3',

miR-214-3p reverse: 5'-CATTCGATCTTCTCCACAG TCTC-3';

NLRP3 forward: 5'-GTGGAGATCCTAGGTTTCTCTG-3', NLRP3 reverse: 5'-CAGGATCTCATTCTCTTGGA TC-3';

caspase-1 forward: 5'-ACACGTCTTGCCCTCATTAT CT-3',

caspase-1 reverse: 5' -ATAACCTTGGGCTTGTCTTT CA-3'

IL-1 $\beta$ forward: $5^{\prime}$-CCCTGCAGCTGGAGAGTGTGG-3' IL-1 $\beta$ reverse: $5^{\prime}$-TGTGCTCTGCTTGAGAGGTGCT-3' GAPDH forward: 5'-ATCACTGCCACCCAGAAGAC-3' GAPDH reverse: 5'-TTTCTAGACGGCAGGTCAGG-3' U6 forward: $5^{\prime}$-CTCGCTTCGGCAGCACATATACT-3' U6 reverse: 5' ${ }^{\prime}$-ACGCTTCACGAATTTGCGTGTC-3'

\section{Protein extraction and western blot analysis}

Total protein samples were extracted and run on 10\% sodium dodecyl sulfate polyacrylamide gel electrophoresis. Proteins were then transferred to nitrocellulose membranes. After blocking with bovine serum albumin (BSA), the membranes were incubated with primary antibodies of NLRP3 (Boster Biological Technology, Wuhan, China, catalog BA3677, 1:800), caspase-1 (Cell Signaling Technology, Danvers, MA, USA, catalog 2225, 1:1000), IL-1 $\beta$ (Cell Signaling Technology, catalog 12703,
1:1000), GSDMD-N (Bioss, Beijing, China, catalog bs14287R 1:1000), collagen I (Abcam, Cambridge, UK, catalog ab34710, 1:800), collagen III (Abcam, catalog ab7778, 1:800), TGF- $\beta 1$ (Cell Signaling Technology, catalog 3711, 1:1000), p-smad2 (Cell Signaling Technology, catalog 3108, 1:1000), smad2 (Cell Signaling Technology, catalog 5339,1:800), p-smad3 (Cell Signaling Technology, catalog 9520, 1:1000), smad3 (Cell Signaling Technology, catalog 9523, 1:1000), and GAPDH (ZSGB-BIO, Beijing, China, catalog TA-08, 1:1000) at $4{ }^{\circ} \mathrm{C}$ overnight followed by the secondary antibody for $1 \mathrm{~h}$. GAPDH was used as internal control. The bands were pictured using GelDox XR System (Bio-Rad, CA, USA). Quantity One software was used to quantify the intensity of the bands.

\section{Immunofluorescence staining}

Fibroblasts were treated with $4 \%$ buffered paraformaldehyde for $20 \mathrm{~min}$ at room temperature. Then, $1 \%$ BSA and $0.1 \%$ Triton-X were used to block the membrane for $2 \mathrm{~h}$ at room temperature and then treated with the primary antibodies against NLRP3, caspase-1, IL- $1 \beta$, collagen I, collagen III and TGF- $\beta 1(1: 200)$ at $4{ }^{\circ} \mathrm{C}$ overnight before treatment with the secondary antibody for $1 \mathrm{~h}$ at room temperature. The nuclei were stained with DAPI (Beyotime, Shanghai, China). The results were captured using a fluorescence microscope. The immunostaining results were quantified and statistically analyzed using Image-Pro Plus 6.0.

\section{Immunohistochemical analysis}

Left ventricle samples were fixed in $4 \%$ paraformaldehyde and embedded in paraffin. Samples were then cut into $5 \mu \mathrm{m}$-thick sections and stained with the primary antibody against NLRP3, caspase-1, IL-1 $\beta$ and GSDMD-N (1:200) at $4{ }^{\circ} \mathrm{C}$ overnight, followed by the secondary antibody. Then, the sections were stained with diaminobenzidine and images were captured by a fluorescence microscope (Nikon 80i, Otawara, Tochigi, Japan).

\section{Luciferase assay}

Both KCNQ1OT1 and caspase-1 $3^{\prime}$-UTRs contain conserved miR-214-3p-binding sites. The mutated $3^{\prime}$ UTRs of KCNQ1OT1 were synthesized by RIBOBIO (Guangzhou, China), and amplified by PCR. The PCR fragment was cloned into the XhoI and NotI sites downstream of the luciferase gene in the psi-CHECK2 vector. The 3'-UTR luciferase vector was co-transfected with miR-214-3p mimics into HEK 293 cells using Lipofectamine 200 (Invitrogen), and Renilla luciferase reporters were used as internal controls. A luciferase activity assay was performed after $48 \mathrm{~h}$ using the Dual-Luciferase Reporter Assay System (Promega Biotech Co., Ltd.) according to the manufacturer's instructions. Similarly, 
wild-type and mutated type CASP1 $3{ }^{\prime}$-UTR were synthesized for use to detect the relationship between CASP1 and miR-214-3p.

\section{Data analysis}

Values were analyzed with Graphpad Prism 6 and were presented as the mean $\pm \mathrm{SD}$. Unpaired student's $t$ tests were performed to compare the differences between the two groups. One-way analysis of variance combined with Bonferroni posttest were performed to compare the differences between the two or different groups. $P<0.05$ was considered statistically significant.

\section{Acknowledgements}

This study was supported by grants from the National Natural Science Foundation of China (81770809 and 81673426), Bethune-Merck Diabetes Research Foundation (G2017044) and Graduate Innovation Fund of Harbin Medical University (YJSCX2017-59HYD).

\section{Author details}

'Department of Endocrinology, The Second Affiliated Hospital of Harbin Medical University, Harbin, China. ${ }^{2}$ Translational Medicine Research and Cooperation Center of Northern China, Heilongjiang Academy of Medical Sciences, Harbin, China. ${ }^{3}$ Department of Pharmacology (State-Province Key Laboratories of iomedicine-Pharmaceutics of China, Key Laboratory of Cardiovascular Medicine Research, Ministry of Education), College of Pharmacy, Harbin Medical University, Harbin, China

\section{Conflict of interest}

The authors declare that they have no conflict of interest.

\section{Publisher's note}

Springer Nature remains neutral with regard to jurisdictional claims in published maps and institutional affiliations.

Supplementary Information accompanies this paper at (https://doi.org/ 10.1038/s41419-018-1029-4).

Received: 2 July 2018 Revised: 13 August 2018 Accepted: 4 September 2018

Published online: 24 September 2018

\section{References}

1. Boudina, S. \& Abel, E. D. Diabetic cardiomyopathy, causes and effects. Rev. Endocr. Metab. Disord. 11, 31-39 (2010).

2. Asbun, J. \& Villarreal, F. J. The pathogenesis of myocardial fibrosis in the setting of diabetic cardiomyopathy. J. Am. Coll. Cardiol. 47, 693-700 (2006).

3. Bergsbaken, T., Fink, S. L. \& Cookson, B. T. Pyroptosis: host cell death and inflammation. Nat. Rev. Microbiol. 7, 99-109 (2009).

4. Schroder, K, Zhou, R. \& Tschopp, J. The NLRP3 inflammasome: a sensor for metabolic danger? Science 327, 296-300 (2010).

5. Sborgi, L. et al. GSDMD membrane pore formation constitutes the mechanism of pyroptotic cell death. EMBO J. 35, 1766-1778 (2016).

6. Shi, J. et al. Cleavage of GSDMD by inflammatory caspases determines pyroptotic cell death. Nature 526, 660-665 (2015).

7. Bracey, N. A. et al. Mitochondrial NLRP3 protein induces reactive oxygen species to promote Smad protein signaling and fibrosis independent from the inflammasome. J. Biol. Chem. 289, 19571-19584 (2014).

8. Jeyabal, P. et al. MicroRNA-9 inhibits hyperglycemia induced cardiac pyroptosis in human ventricular cardiomyocytes by targeting ELAVL1. Biochem. Biophys. Res. Commun. 471, 423-429 (2016).
9. Li, X. et al. MicroRNA-30d regulates cardiomyocyte pyroptosis by directly targeting foxo3a in diabetic cardiomyopathy. Cell Death Dis. 5, e1479 (2014).

10. Luo, B. et al. NLRP3 gene silencing ameliorates diabetic cardiomyopathy in a type 2 diabetes rat model. PLOS ONE 9, e104771 (2014).

11. Luo, B. et al. Rosuvastatin alleviates diabetic cardiomyopathy by inhibiting NLRP3 inflammasome and MAPK pathways in a type 2 diabetes rat model. Cardiovasc. Drugs Ther. 28, 33-43 (2014).

12. Ponting, C. P., Oliver, P. L. \& Reik, W. Evolution and functions of long noncoding RNAs. Cell 136, 629-641 (2009).

13. Wang, K. C. \& Chang, H. Y. Molecular mechanisms of long noncoding RNAs. Mol. Cell 43, 904 (2011).

14. Gao, X., Ge, J., Li, W., Zhou, W. \& Xu, L. LncRNA KCNQ1OT1 ameliorates particleinduced osteolysis through inducing macrophage polarization by inhibiting miR-21a-5p. Biol. Chem. 399, 375-386 (2018).

15. Coto, E. et al. Differential methylation of IncRNA KCNQ1OT1 promoter polymorphism was associated with symptomatic cardiac long QT. Epigenomics $\mathbf{9}$, 1049-1057 (2017)

16. Li, X. et al. Down-regulation of IncRNA KCNQ1OT1 protects against myocardial ischemia/reperfusion injury following acute myocardial infarction. Biochem. Biophys. Res. Commun. 491, 1026-1033 (2017).

17. Jin, X., Jin, H., Shi, Y., Guo, Y. \& Zhang, H. Long non-coding RNA KCNQ1OT1 promotes cataractogenesis via miR-214 and activation of the caspasepathway. Cell Physiol. Biochem. 42, 295-305 (2017).

18. Ren, K., Xu, R., Huang, J., Zhao, J. \& Shi, W. Knockdown of long non-coding RNA KCNQ1OT1 depressed chemoresistance to paclitaxel in lung adenocarcinoma. Cancer Chemother. Pharmacol. 80, 243-250 (2017).

19. Guo, B., Zhang, Q., Wang, H., Chang, P. \& Tao, K. KCNQ1OT1 promotes melanoma growth and metastasis. Aging 10, 632-644 (2018).

20. Asahara, S. et al. Paternal allelic mutation at the Kcnq1 locus reduces pancreatic beta-cell mass by epigenetic modification of Cdkn1c. Proc. Natl. Acad. Sci. USA 112, 8332-8337 (2015).

21. Wilusz, J. E., Sunwoo, H. \& Spector, D. L. Long noncoding RNAs: functional surprises from the RNA world. Genes Dev. 23, 1494-1504 (2009).

22. Rinn, J. L. \& Chang, H. Y. Genome regulation by long noncoding RNAs. Annu. Rev. Biochem. 81, 145-166 (2012).

23. Miao, E. A. et al. Caspase-1-induced pyroptosis is an innate immune effector mechanism against intracellular bacteria. Nat. Immunol. 11, 1136-1142 (2010).

24. Artlett, C. M. The role of the NLRP3 inflammasome in fibrosis. Open Rheumatol. J. 6, 80-86 (2012)

25. Wang, Y., Wu, Y., Chen, J., Zhao, S. \& Li, H. Pirfenidone attenuates cardiac fibrosis in a mouse model of TAC-induced left ventricular remodeling by suppressing NLRP3 inflammasome formation. Cardiology 126, 1-11 (2013).

26. Fernández, L. \& Mosquera, J. A. Interleukin-1 increases fibronectin production by cultured rat cardiac fibroblasts. Pathobiology 70, 191-196 (2002).

27. Luo, D. D., Fielding, C., Phillips, A. \& Fraser, D. Interleukin-1 beta regulates proximal tubular cell transforming growth factor beta-1 signalling. Nephrol. Dial. Transplant. 24, 2655-2665 (2009).

28. Chaudhuri, V., Zhou, L. \& Karasek, M. Inflammatory cytokines induce the transformation of human dermal microvascular endothelial cells into myofibroblasts: a potential role in skin fibrogenesis. J. Cutan. Pathol. 34, 146-153 (2007).

29. Postlethwaite, A. E. et al. Modulation of fibroblast functions by interleukin-1: increased steady-state accumulation of type I procollagen messenger RNAs and stimulation of other functions but not chemotaxis by human recombinant interleukin-1 alpha and beta. J. Cell Biol. 106, 311-318 (1988).

30. Liu, H. J. et al. Apigenin alleviates STZ-induced diabetic cardiomyopathy. Mol Cell Biochem. 428, 1-13 (2017).

31. Jiang, $Y$. et al. Downregulation of long non-coding RNA Kcnq1ot1: an important mechanism of arsenic trioxide-induced long QT syndrome. Cell. Physiol. Biochem. 45, 192-202 (2018).

32. Yang, Z. et al. Deletion of interleukin-6 alleviated interstitial fibrosis in streptozotocin-induced diabetic cardiomyopathy of mice through affecting TGFß1 and miR-29pathways. Sci. Rep. 6, 23010 (2016).

33. Ma, T. et al. Microarray analysis of differentially expressed microRNAs in nonregressed and regressed bovine corpus luteum tissue; microRNA-378 may suppress luteal cell apoptosis by targeting the interferon gamma receptor 1 gene. J. Appl. Genet. 52, 481-486 (2011). 